\title{
Secure Italian domination in graphs
}

\author{
M. Dettlaff ${ }^{1}\left(\mathbb{D} \cdot\right.$ M. Lemańska ${ }^{1}$. J. A. Rodríguez-Velázquez ${ }^{2}$
}

Accepted: 29 September 2020 / Published online: 28 October 2020

(c) The Author(s) 2020

\section{Abstract}

An Italian dominating function (IDF) on a graph $G$ is a function $f: V(G) \rightarrow$ $\{0,1,2\}$ such that for every vertex $v$ with $f(v)=0$, the total weight of $f$ assigned to the neighbours of $v$ is at least two, i.e., $\sum_{u \in N_{G}(v)} f(u) \geq 2$. For any function $f: V(G) \rightarrow\{0,1,2\}$ and any pair of adjacent vertices with $f(v)=0$ and $u$ with $f(u)>0$, the function $f_{u \rightarrow v}$ is defined by $f_{u \rightarrow v}(v)=1, f_{u \rightarrow v}(u)=f(u)-1$ and $f_{u \rightarrow v}(x)=f(x)$ whenever $x \in V(G) \backslash\{u, v\}$. A secure Italian dominating function on a graph $G$ is defined as an IDF $f$ which satisfies that for every vertex $v$ with $f(v)=0$, there exists a neighbour $u$ with $f(u)>0$ such that $f_{u \rightarrow v}$ is an IDF. The weight of $f$ is $\omega(f)=\sum_{v \in V(G)} f(v)$. The minimum weight among all secure Italian dominating functions on $G$ is the secure Italian domination number of $G$. This paper is devoted to initiating the study of the secure Italian domination number of a graph. In particular, we prove that the problem of finding this parameter is NP-hard and we obtain general bounds on it. Moreover, for certain classes of graphs, we obtain closed formulas for this novel parameter.

Keywords Domination number - Secure domination number - Italian domination . Secure Italian domination

Mathematics Subject Classification 05C69

$\bowtie \quad$ M. Dettlaff

magda.dettlaff1@pg.edu.pl

M. Lemańska

magdalena.lemanska@pg.edu.pl

J. A. Rodríguez-Velázquez

juanalberto.rodriguez@urv.cat

1 Department of Technical Physics and Applied Mathematics, Gdansk University of Technology, ul. Narutowicza 11/12, 80-233 Gdańsk, Poland

2 Departament d'Enginyeria Informàtica i Matemàtiques, Universitat Rovira i Virgili, Av. Països Catalans 26, 43007 Tarragona, Spain 


\section{Introduction}

The following approach to protection of a graph was described by Cockayne et al. (2005). Suppose that one or more guards are stationed at some of the vertices of a simple graph $G$ and that a guard at a vertex can deal with a problem at any vertex in its closed neighbourhood. Consider a function $f: V(G) \longrightarrow\{0,1,2\}$ where $f(v)$ is the number of guards at $v$, and let $V_{i}=\{v \in V(G): f(v)=i\}$ for every $i \in\{0,1,2\}$. We will identify a function $f$ with the subsets $V_{0}, V_{1}, V_{2}$ of $V(G)$ associated with it, and so we will use the unified notation $f\left(V_{0}, V_{1}, V_{2}\right)$ for the function and these associated subsets. The weight of $f$ is defined to be $\omega(f)=f(V(G))=\left|V_{1}\right|+2\left|V_{2}\right|$. Informally, we say that $G$ is protected under $f$ if there is at least one guard available to handle a problem at any vertex. Next we show some approaches to the protection of graphs. The functions in each approach protect the graph according to a certain strategy.

We assume that the reader is familiar with the basic concepts, notation and terminology of domination in graphs. If this is not the case, we suggest the textbooks (Haynes et al. 1998a, b). For the remainder of the paper, definitions will be introduced whenever a concept is needed.

A Roman dominating function (RDF) is a function $f\left(V_{0}, V_{1}, V_{2}\right)$ such that for every vertex $v \in V_{0}$ there exists a vertex $u \in N_{G}(v) \cap V_{2}$, where $N_{G}(v)$ denotes the open neighbourhood of $v$. The Roman domination number, denoted by $\gamma_{R}(G)$, is the minimum weight among all RDFs on $G$. This concept of protection has historical motivation (Stewart 1999) and was formally proposed by Cockayne et al. (2004). A Roman dominating function with minimum weight $\gamma_{R}(G)$ on $G$ is called a $\gamma_{R}(G)$ function. A similar agreement will be assumed when referring to optimal functions (and sets) associated with other parameters used in the article.

A generalization of Roman domination, known as Italian domination, was introduced by Chellali et al. (2016) under the name of Roman \{2\}-domination. The concept was studied further in Henning and Klostermeyer (2017) and Klostermeyer and MacGillivray (2019). An Italian dominating function (IDF) on a graph $G$ is a function $f\left(V_{0}, V_{1}, V_{2}\right)$ satisfying that $f\left(N_{G}(v)\right)=\sum_{u \in N_{G}(v)} f(u) \geq 2$ for every $v \in V_{0}$, i.e., $f\left(V_{0}, V_{1}, V_{2}\right)$ is an IDF if $N_{G}(v) \cap V_{2} \neq \emptyset$ or $\left|N_{G}(v) \cap V_{1}\right| \geq 2$ for every $v \in V_{0}$. The Italian domination number, denoted by $\gamma_{I}(G)$, is the minimum weight among all IDFs on $G$. Since every $\gamma_{R}(G)$-function is an IDF, $\gamma_{R}(G) \geq \gamma_{I}(G)$.

For any function $f\left(V_{0}, V_{1}, V_{2}\right)$ and any pair of adjacent vertices $v \in V_{0}$ and $u \in$ $V_{1} \cup V_{2}$, the function $f_{u \rightarrow v}$ is defined by $f_{u \rightarrow v}(v)=1, f_{u \rightarrow v}(u)=f(u)-1$ and $f_{u \rightarrow v}(x)=f(x)$ whenever $x \in V(G) \backslash\{u, v\}$.

A vertex $v \in V_{0}$ is said to be undefended under a function $f\left(V_{0}, V_{1}, V_{2}\right)$ if $N_{G}(v) \cap$ $\left(V_{1} \cup V_{2}\right)=\varnothing$. A function $f\left(V_{0}, V_{1}, V_{2}\right)$ is a weak Roman dominating function (WRDF) if for every vertex $v \in V_{0}$ there exists $u \in N(v) \cap\left(V_{1} \cup V_{2}\right)$ such that $G$ does not have undefended vertices under $f_{u \rightarrow v}$. The weak Roman domination number, denoted by $\gamma_{r}(G)$, is the minimum weight among all WRDFs on $G$. This concept of protection was introduced by Henning and Hedetniemi (2003) and studied further in Chellali et al. (2014), Cockayne et al. (2003) and Valveny et al. (2019).

Notice that, every $\gamma_{I}(G)$-function is a WRDF, which implies that $\gamma_{I}(G) \geq \gamma_{r}(G)$. 
A secure dominating set is a dominating set $S$ which satisfies that for every $v \in$ $V(G) \backslash S$ there exists $u \in S \cap N_{G}(v)$ such that $(S \backslash\{u\}) \cup\{v\}$ is a dominating set as well. The secure domination number, denoted by $\gamma_{S}(G)$, is the minimum cardinality among all secure dominating sets. Notice that $S$ is a secure dominating set if and only if there exists a WRDF $f\left(V_{0}, V_{1}, V_{2}\right)$ such that $V_{1}=S$ and $V_{2}=\emptyset$. Hence, $\gamma_{s}(G) \geq \gamma_{r}(G)$. This concept of protection was introduced by Cockayne et al. (2005), and studied further in Merouane and Chellali (2015), Burger et al. (2008), Chellali et al. (2014), Cockayne et al. (2003), Klostermeyer and Mynhardt (2008) and Valveny and Rodríguez-Velázquez (2019).

Now, from the previous inequalities, we derive the following inequality chains.

$$
\gamma_{R}(G) \geq \gamma_{I}(G) \geq \gamma_{r}(G) \geq \gamma(G) \text { and } \gamma_{s}(G) \geq \gamma_{r}(G) \geq \gamma(G)
$$

In this article we introduce the study of secure Italian domination in graphs. We define a secure Italian dominating function (SIDF) to be an IDF $f\left(V_{0}, V_{1}, V_{2}\right)$ which satisfies that for every vertex $v \in V_{0}$ there exists $u \in N_{G}(v) \cap\left(V_{1} \cup V_{2}\right)$ such that the $f_{u \rightarrow v}$ is an IDF on $G$. In particular, whenever $f_{u \rightarrow v}$ is an IDF, we will say that $u$ is a moving neighbour of $v$. Obviously, if $f\left(V_{0}, V_{1}, V_{2}\right)$ is an SIDF, then every vertex $v \in V_{0}$ has at least one moving neighbour. The secure Italian domination number, denoted by $\gamma_{I}^{S}(G)$, is the minimum weight among all SIDFs on $G$. In Fig. 1 we show two examples of $\gamma_{I}^{s}(G)$-functions.

By definition of secure Italian domination number we immediately deduce the following inequalities.

$$
\gamma_{I}^{S}(G) \geq \gamma_{I}(G) \text { and } \gamma_{I}^{S}(G) \geq \gamma_{r}(G)
$$

The paper is structured as follows. In Sect. 2 we show that the general problem of finding the secure Italian domination number of a graph is NP-hard. In Sect. 3 we derive general bounds and discuss some extremal cases. In particular, we show that any non-empty graph $G$ satisfies $\max \left\{\gamma_{2}(G), \gamma_{r}(G)+1\right\} \leq \gamma_{I}^{s}(G) \leq \gamma(G)+\gamma_{s}(G)$. In Sect. 4 we obtain a formula for the secure Italian domination number of paths and
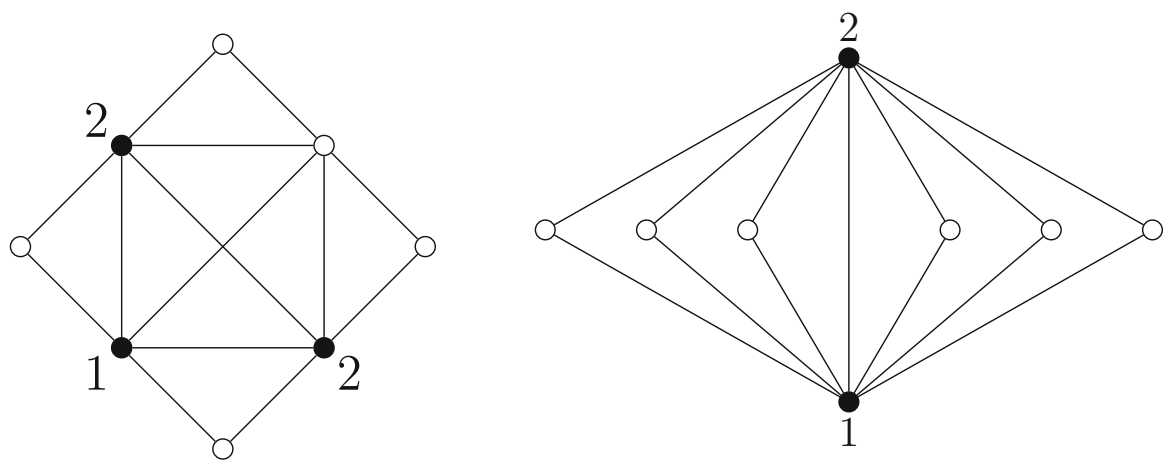

Fig. 1 The labels in these graphs define $\gamma_{I}^{S}(G)$-functions 
cycles. Furthermore, we characterize all trees $T$ satisfying $\gamma_{I}^{s}(T)=\gamma(T)+1$ or $\gamma_{I}^{S}(T)=\gamma(T)+2$. Finally, Sect. 5 is devoted to the study of join graphs.

\section{NP-hardness}

This section is devoted to prove that for any graph $G$ of order $n$ there exists a graph $G^{\prime}$ such that $\gamma_{I}^{S}\left(G^{\prime}\right)=\gamma_{r}(G)+n$, showing that the problem of finding $\gamma_{I}^{S}\left(G^{\prime}\right)$ is as difficult as the problem of finding $\gamma_{r}(G)$. To begin with, we need to state the following lemma. Let $L(G)$ denotes the set of all leaves in $G$.

Lemma 1 If $G$ is a graph with set of leaves $L(G) \neq \emptyset$, then there exists a $\gamma_{I}^{s}(G)$ function $f$ such that $f(v)=1$ for every $v \in L(G)$.

Proof Let $f$ be a $\gamma_{I}^{s}(G)$-function, $v \in L(G)$ and $s$ the support of $v$. If $f(v)=2$, then $f(s)=0$, by the minimality of $\omega(f)$. In such a case, $f^{\prime}=f_{v \rightarrow s}$ is a $\gamma_{I}^{s}(G)$-function with $f^{\prime}(v)=1$. Now, if $f(v)=0$, then $f(s)=2$ and $s$ is a moving neighbour only of $v$. Hence, $f^{\prime \prime}=f_{s \rightarrow v}$ is a $\gamma_{I}^{s}(G)$-function with $f^{\prime \prime}(v)=1$. Therefore, the result follows.

Let $G$ be a graph with vertex set $V(G)=\left\{v_{1}, \ldots, v_{n}\right\}$ and let $H$ be a rooted graph. The rooted product of $G$ and $H$ is the graph $G \circ H$ constructed from $G$ and $n$ copies $H_{1}, H_{2}, \ldots, H_{n}$ of $H$, by identifying $v_{i}$ with the root vertex of $H_{i}$ for every $i \in\{1, \ldots, n\}$.

Theorem 2 For any graph $G$ of order $n, \gamma_{I}^{s}\left(G \circ K_{2}\right)=n+\gamma_{r}(G)$.

Proof Let $h$ be a $\gamma_{r}(G)$-function and $h^{\prime}$ the function defined on $G \circ K_{2}$ in the way that $h^{\prime}(x)=h(x)$ if $x \in V(G)$ and $h^{\prime}(x)=1$ otherwise. Since $h^{\prime}$ is an SIDF on $G \circ K_{2}$, we conclude that $\gamma_{I}^{S}\left(G \circ K_{2}\right) \leq \omega\left(h^{\prime}\right)=n+\omega(h)=n+\gamma_{r}(G)$.

We show that $\gamma_{I}^{s}\left(G \circ K_{2}\right) \geq n+\gamma_{r}(G)$. Let $f\left(V_{0}, V_{1}, V_{2}\right)$ be a $\gamma_{I}^{s}\left(G \circ K_{2}\right)$-function. By Lemma 1 we can assume that $f(v)=1$ for every leaf $v$. Let $g\left(W_{0}, W_{1}, W_{2}\right)$ be the restriction of $f$ to $V(G)$. That is, $W_{i}=V_{i} \cap V(G)$ for $i \in\{0,1,2\}$. Notice that $W_{0}=V_{0}$ and $W_{2}=V_{2}$. We claim that $g$ is a WRDF on $G$. We have to show that for every $x \in W_{0}$ there exists $y \in W_{1} \cup W_{2}$ such that $G$ does not have undefended vertex under $g_{y \rightarrow x}$. We know that for every $x \in V_{0}=W_{0}$ there exists $y \in V_{1} \cup V_{2}$ such that $f_{y \rightarrow x}\left(V_{0}^{\prime}, V_{1}^{\prime}, V_{2}^{\prime}\right)$ is an IDF on $G \circ K_{2}$, and also the restriction of $f_{y \rightarrow x}$ to $V(G)$ equals $g_{y \rightarrow x}$. Thus, $y$ has to belong to $W_{1} \cup W_{2}$ and for every $z \in V_{0} \backslash\{x\}$ either $\left|N_{G \circ K_{2}}(z) \cap V_{1}^{\prime}\right| \geq 2$ or $\left|N_{G \circ K_{2}}(z) \cap V_{2}^{\prime}\right| \geq 1$, which implies that $\left|N_{G}(z) \cap V_{1}^{\prime} \cap V(G)\right| \geq$ 1 or $\left|N_{G}(z) \cap V_{2}^{\prime} \cap V(G)\right| \geq 1$. Consequently, $G$ does not have undefended vertex under $g_{y \rightarrow x}$. Therefore, $\gamma_{I}^{s}\left(G \circ K_{2}\right)=\omega(f)=\left|V_{1}\right|+2\left|V_{2}\right|=n+\left|W_{1}\right|+2\left|W_{2}\right|=$ $n+\omega(g) \geq n+\gamma_{r}(G)$.

Our next result shows that we can use rooted product graphs to study the problem of finding the secure Italian domination number of a graph. In this case, the main tool is Theorem 2. It is well known that the weak Roman dominating set problem is an NP-complete decision problem (Henning and Hedetniemi 2003), i.e., given a positive integer $k$ and a graph $G$, the problem of deciding if $G$ has a weak Roman dominating 
set $D$ of cardinality $|D| \leq k$ is NP-complete. Hence, the optimization problem of computing the weak Roman domination number of a graph is NP-hard.

Corollary 3 The problem of computing the secure Italian domination number of a graph is NP-hard.

Proof By Theorem 2, for any graph $G$ of order $n$ we have that $\gamma_{I}^{s}\left(G \circ K_{2}\right)=n+$ $\gamma_{r}(G)$. Hence, the problem of computing $\gamma_{r}(G)$ is equivalent to the problem of finding $\gamma_{I}^{s}\left(G \circ K_{2}\right)$, which implies that the general problem of computing the secure Italian domination number of a graph is NP-hard.

According to Corollary 3, it would be desirable to obtain tight bound or closed formulas for the secure Italian domination number of a graph. This is precisely the aim of the next sections.

\section{General bounds and extremal cases}

To begin this section, we proceed to characterize the graphs achieving the following trivial bounds.

Remark 4 For any graph $G$ of order $n \geq 2$,

$$
2 \leq \gamma_{I}^{s}(G) \leq n
$$

Obviously, when characterizing the graphs achieving the bounds we can restrict ourselves to the case of connected non-trivial graphs, and from that characterization it is easy to deduce the result for non-connected graphs.

Theorem 5 Let $G$ be a connected non-trivial graph of order $n$. Then the following statements hold.

(i) $\gamma_{I}^{s}(G)=2$ if and only if $G \cong K_{n}$;

(ii) $\gamma_{I}^{s}(G)=n$ if and only if $G \cong K_{1, n-1}$.

Proof If $G \cong K_{n}$, then obviously $\gamma_{I}^{s}(G)=2$. Suppose $\gamma_{I}^{s}(G)=2$ and $G \nVdash K_{n}$. Let $f$ be a $\gamma_{I}^{s}(G)$-function and $u, v$ two non-adjacent vertices. If $f(u)=2$, then $f\left(N_{G}[v]\right)=0$, which is a contradiction. Assume that $f(u)=0$. Hence, either $u$ is adjacent to two vertices $x, y$ where $f(x)=f(y)=1$ or $u$ is adjacent to a vertex $z$ such that $f(z)=2$. Thus, since $\gamma_{I}^{s}(G)=2$, in both cases $f(v)=0$. In the first case, neither $f_{x \rightarrow u}$ nor $f_{y \rightarrow u}$ is an IDF on $G$, which is a contradiction. In the second case, $f_{z \rightarrow u}$ is not an IDF on $G$, which is a contradiction again. Now, if $f(u)=f(v)=1$, then for any vertex $x \in V(G) \backslash\{u, v\}=N_{G}(u) \cap N_{G}(v)$ we have that $f(x)=0$ and neither $f_{u \rightarrow x}$ nor $f_{v \rightarrow x}$ is an IDF on $G$, which is a contradiction again. Therefore, (i) follows.

If $G \cong K_{1, n-1}$, then it is easy to observe that $\gamma_{I}^{S}(G)=n$. Conversely, suppose $\gamma_{I}^{S}(G)=n$ and there are two vertices, $u, v$ such that $\mathrm{d}_{G}(u) \geq 2$ and $\mathrm{d}_{G}(v) \geq 2$; let us choose $u, v$ such that $u v \in E(G)$. We can define an SIDF $g$ on $G$ such that $g(u)=0$ and $g(x)=1$ for any $x \in V(G) \backslash\{u\}$. Notice that $\omega(g)=n-1<n=\gamma_{I}^{s}(G)$, which is a contradiction. Therefore, (ii) follows. 
For any vertex $x \in V(G)$ and any $\gamma_{I}^{s}(G)$-function $f\left(V_{0}, V_{1}, V_{2}\right)$ such that $f(x)=$ 2, we define the following set associated with $x$ and $f$,

$$
P_{f}(x)=\left\{y \in V_{0}: N_{G}(y) \cap\left(V_{1} \cup V_{2}\right)=\{x\}\right\} .
$$

The subgraph of $G$ induced by a set $S \subseteq V(G)$ will be denoted by $\langle S\rangle$. From the definition of SIDF on $G$ we have the following straightforward observation.

Observation 6 If there exists a vertex $x \in V(G)$ and a $\gamma_{I}^{s}(G)$-function $f$ such that $f(x)=2$ and $P_{f}(x) \neq \emptyset$, then $\left\langle P_{f}(x)\right\rangle$ is a clique.

The $k$-domination number of a graph $G$, denoted by $\gamma_{k}(G)$, is the cardinality of a smallest set of vertices such that every vertex not in the set is adjacent to at least $k$ vertices in the set. Such sets are called $k$-dominating sets. Since every 2-dominating set $S$ is a secure dominating set, and the function $f\left(V_{0}, V_{1}=S, V_{2}=\emptyset\right)$ is an IDF, we conclude that

$$
\gamma_{2}(G) \geq \gamma_{s}(G) \text { and } \gamma_{2}(G) \geq \gamma_{I}(G)
$$

From the inequality $\gamma_{2}(G) \geq \gamma_{s}(G)$ and the lower bound stated by the next theorem, we will be able to deduce that the expected inequality $\gamma_{I}^{S}(G) \geq \gamma_{s}(G)$ holds for any graph $G$.

Theorem 7 For any non-empty graph $G$,

$$
\max \left\{\gamma_{2}(G), \gamma_{r}(G)+1\right\} \leq \gamma_{I}^{s}(G) \leq \gamma(G)+\gamma_{s}(G)
$$

Proof Let $D$ be a $\gamma(G)$-set and $D^{\prime}$ a $\gamma_{s}(G)$-set. Let us construct a function $f$ from $D$ and $D^{\prime}$ such that

$$
f(x)= \begin{cases}2 & \text { if } x \in D \cap D^{\prime} \\ 1 & \text { if } x \in\left(D \cup D^{\prime}\right) \backslash\left(D \cap D^{\prime}\right), \\ 0 & \text { otherwise }\end{cases}
$$

We claim that $f$ is an SIDF on $G$. Obviously, $f$ is an IDF on $G$. For every vertex $x \in V(G) \backslash\left(D \cup D^{\prime}\right)$ there exists $y \in D^{\prime}$ such that $x$ is protected by $y$ under $D^{\prime}$. We will show that $f_{y \rightarrow x}$ is an IDF on $G$. Let $x^{\prime}$ be a vertex such that $f_{y \rightarrow x}\left(x^{\prime}\right)=0$. If $x^{\prime} \notin N_{G}(y)$, then we are done as $f$ is an IDF on $G$. Assume $x^{\prime} \in N_{G}[y]$. We consider two cases.

Case $1 x^{\prime} \in N_{G}(x)$. If $y \in D \cap D^{\prime}$, then $f_{y \rightarrow x}(x)=f_{y \rightarrow x}(y)=1$. Otherwise there exists $z \in N_{G}\left(x^{\prime}\right) \cap D$ such that $f_{y \rightarrow x}(x)=f_{y \rightarrow x}(z)=1$.

Case $2 x^{\prime} \notin N_{G}(x)$. In this case, there exists $y^{\prime} \in\left(D^{\prime} \backslash\{y\}\right) \cap N_{G}\left(x^{\prime}\right)$ and $z \in$ $N_{G}\left(x^{\prime}\right) \cap D$. If $y^{\prime}=z$, then $f_{y \rightarrow x}\left(y^{\prime}\right)=2$, otherwise $f_{y \rightarrow x}\left(y^{\prime}\right) \geq 1$ and $f_{y \rightarrow x}(z) \geq 1$.

According to the two cases above we can conclude that $f_{y \rightarrow x}$ is an IDF on $G$. Therefore, $f$ is an SIDF, and so $\gamma_{I}^{s}(G) \leq \omega(f)=|D|+\left|D^{\prime}\right|=\gamma(G)+\gamma_{s}(G)$.

Now we prove the lower bound $\gamma_{I}^{s}(G) \geq \gamma_{2}(G)$. Let $g\left(V_{0}, V_{1}, V_{2}\right)$ be a $\gamma_{I}^{s}(G)$ function. Notice that if $V_{2}=\emptyset$, then $V_{1}$ is a 2-dominating set and so $\gamma_{2}(G) \leq$ 
$\left|V_{1}\right|=\gamma_{I}^{s}(G)$. Assume that $V_{2} \neq \emptyset$. Let $x \in V_{2}$ and notice that if $P_{g}(x) \neq \emptyset$, then the subgraph induced by $P_{g}(x)$ is a clique. With this fact in mind, for every $x \in V_{2}$ such that $P_{g}(x) \neq \emptyset$ we fix one vertex $x^{\prime} \in P_{g}(x)$, and let $V_{g}$ be the set of these representatives. Now, if $u \in V_{0}$ and $u$ does not belong to any $P_{g}(x)$, then $\left|N_{G}(u) \cap\left(V_{1} \cup V_{2}\right)\right| \geq 2$, while if $u \in P_{g}(x)$ for some $x \in V_{2}$, then $x, x^{\prime} \in N_{G}[u]$. Thus $S=V_{1} \cup V_{2} \cup V_{g}$ is a 2-dominating set of $G$. Therefore, $\gamma_{2}(G) \leq|S|=\left|V_{1}\right|+\left|V_{2}\right|+\left|V_{g}\right| \leq\left|V_{1}\right|+2\left|V_{2}\right|=$ $\gamma_{I}^{S}(G)$ and the lower bound $\gamma_{I}^{s}(G) \geq \gamma_{2}(G)$ follows.

Finally, we proceed to prove the lower bound $\gamma_{I}^{s}(G) \geq \gamma_{r}(G)+1$. In this case, let $h\left(W_{0}, W_{1}, W_{2}\right)$ be a $\gamma_{I}^{s}(G)$-function. If $\gamma_{I}^{s}(G)=|V(G)|$, then we are done, as $\gamma_{r}(G) \leq|V(G)|-1$ for every non-empty graph. Hence, we fix $x \in W_{0}$ and $y \in W_{1} \cup W_{2}$ such that $y$ is a moving neighbour of $x$. We now construct a weak Roman dominating function $h^{\prime}\left(W_{0}^{\prime}, W_{1}^{\prime}, W_{2}^{\prime}\right)$, which is defined in two different ways depending on whether $y \in W_{2}$ or not.

Case $\mathbf{1}^{\prime} y \in W_{2}$. In this case, we define the function $h^{\prime}$ by $h^{\prime}(y)=1$ and $h^{\prime}(z)=$ $h(z)$ for every $z \in V(G) \backslash\{y\}$. Since $h$ is an IDF, $G$ does not have undefended vertices under $h^{\prime}$, i.e., $W_{1} \cup W_{2}=W_{1}^{\prime} \cup W_{2}^{\prime}$ is a dominating set. Moreover, since for every $v \in W_{0}=W_{0}^{\prime}$, there exits $u \in W_{1} \cup W_{2}$ such that $h_{u \rightarrow v}$ is an IDF, we conclude that $G$ does not have undefended vertices under $h_{u \rightarrow v}^{\prime}$. Therefore, $h^{\prime}$ is a weak Roman dominating function on $G$.

Case $2^{\prime} y \in W_{1}$. In this case, we define the function $h^{\prime}$ by $W_{0}^{\prime}=W_{0} \cup\{y\}$, $W_{1}^{\prime}=W_{1} \backslash\{y\}$ and $W_{2}^{\prime}=W_{2}$. Since $h$ and $h_{y \rightarrow x}$ are IDFs, we can conclude that $G$ does not have undefended vertices under $h^{\prime}$. Moreover, for every $v \in W_{0}$, there exits $u \in W_{1} \cup W_{2}$ such that $h_{u \rightarrow v}$ is an IDF. Hence, if $u \neq y$, then $G$ does not have undefended vertices under $h_{u \rightarrow v}^{\prime}$. Suppose that $y$ is the only moving neighbour of $v \in W_{0}$ under $h$ and there exists $w \in W_{0}^{\prime}$ which is undefended under the function $h_{x^{\prime} \rightarrow v}^{\prime}$, where $x^{\prime} \in N_{G}(v) \cap\left(W_{1} \cup W_{2}\right) \backslash\{y\}$. In such a case, $h^{\prime}\left(x^{\prime}\right)=h\left(x^{\prime}\right)=1$, $w \notin N_{G}(v)$ and $N_{G}(w) \cap\left(W_{1} \cup W_{2}\right)=\left\{x^{\prime}, y\right\}$. Thus, $h_{y \rightarrow v}\left(N_{G}(w)\right)=h\left(x^{\prime}\right)=1$, which is a contradiction. Finally, it is readily seen that the set $X=N_{G}(y) \cap\left(W_{1} \cup W_{2}\right)$ is not empty and $G$ does not have undefended vertices under the function $h_{z \rightarrow y}^{\prime}$ for every $z \in X$. Therefore, $h^{\prime}$ is a weak Roman dominating function on $G$.

According to the two cases above, $\gamma_{I}^{s}(G)=\omega(h)=\omega\left(h^{\prime}\right)+1 \geq \gamma_{r}(G)+1$. Therefore, the proof is complete.

Next we show that the bounds above are tight.

- If $G$ is the graph shown in Fig. 2 or the graph shown in Fig. 1 on the right, then $\gamma_{I}^{s}(G)=\gamma_{r}(G)+1$.

- If $G \cong K_{1, n-1}, G \cong K_{n}$ or $G$ is the graph shown in Fig. 1 on the left, then $\gamma_{I}^{s}(G)=\gamma(G)+\gamma_{s}(G)$.

- If $G$ is a corona graph $H \odot K_{p}$ for $p \geq 2$, then $\gamma_{s}(G)=\gamma(G)=|V(H)|$ and $\gamma_{2}(G)=2|V(H)|$, which implies that $\gamma_{2}(G)=\gamma_{I}^{s}(G)=\gamma_{s}(G)+\gamma(G)$.

In summary, we can state the following domination chains.

Remark 8 For any non-empty graph $G$,

(a) $\gamma_{r}(G) \leq \min \left\{\gamma_{I}(G), \gamma_{s}(G)\right\} \leq \max \left\{\gamma_{I}(G), \gamma_{s}(G)\right\} \leq \gamma_{2}(G) \leq \gamma_{I}^{s}(G) \leq$ $\gamma(G)+\gamma_{s}(G)$. 


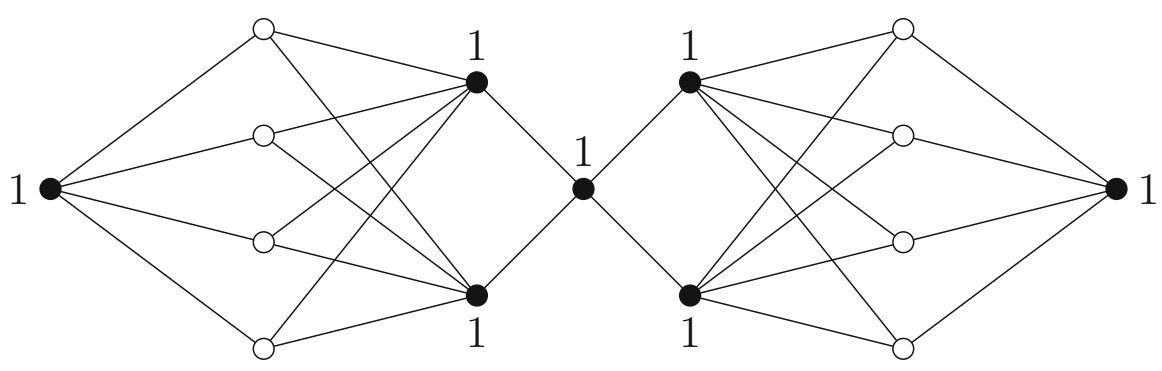

Fig. 2 A graph with $\gamma_{I}^{s}(G)=\gamma_{r}(G)+1=7$ and $\gamma_{2}(G)=\gamma_{I}(G)=\gamma_{s}(G)=\gamma_{r}(G)=6$

(b) $\gamma_{r}(G)+\left|\gamma_{I}(G)-\gamma_{s}(G)\right| \leq \gamma_{2}(G) \leq \gamma_{I}^{s}(G) \leq \gamma(G)+\gamma_{s}(G)$.

(c) $\gamma(G)+1 \leq \gamma_{r}(G)+1 \leq \gamma_{I}^{s}(G) \leq \gamma(G)+\gamma_{s}(G)$.

For any set $S \subseteq V(G)$ and any pair of different vertices $x, y \in S$, we define the following set.

$$
S_{x y}=\left\{v \in V(G) \backslash S: N_{G}(v) \cap S=\{x, y\}\right\} .
$$

Theorem 9 If for every $\gamma_{2}(G)$-set $S$ there exist two vertices $x, y \in S$ such that the set $S_{x y}$ contains two non-adjacent vertices, then

$$
\gamma_{I}^{S}(G) \geq \gamma_{2}(G)+1
$$

Proof We claim that if $\gamma_{I}^{S}(G)=\gamma_{2}(G)$, then there exists a $\gamma_{2}(G)$-set $S$ such that for every $x, y \in S$ either $S_{x y}=\emptyset$ or $\left\langle S_{x y}\right\rangle$ is a clique.

Let $g\left(V_{0}, V_{1}, V_{2}\right)$ be a $\gamma_{I}^{S}(G)$-function and $S$ the 2-dominating set constructed in the proof of Theorem 7, i.e., if $V_{2}=\emptyset$, then $S=V_{1}$, while if $V_{2} \neq \emptyset$, then $S=V_{1} \cup V_{2} \cup V_{g}$. Since $\gamma_{I}^{S}(G)=\gamma_{2}(G)$, we have that $S$ is a $\gamma_{2}(G)$-set. Suppose to the contrary that there exist two different vertices $x, y \in S$ and two different nonadjacent vertices $u, v \in V_{0}$ such that $u, v \in S_{x, y}$. We differentiate the following cases.

Case $1 x, y \in V_{1}$. Since $u$ and $v$ are not adjacent, neither $g_{x \rightarrow u}$ nor $g_{y \rightarrow u}$ is an IDF, which is a contradiction, as $g$ is a $\gamma_{I}^{S}(G)$-function.

Case $2 x \in V_{1}$ and $y \in V_{2}$. Since $\left\langle S_{x y}\right\rangle$ is not a clique and $g$ is a $\gamma_{I}^{s}(G)$-function, $P_{g}(y)=\emptyset$. Hence, $\left|V_{g}\right|<\left|V_{2}\right|$, which implies that $\gamma_{2}(G)=|S|=\left|V_{1}\right|+\left|V_{2}\right|+\left|V_{g}\right|<$ $\left|V_{1}\right|+2\left|V_{2}\right|=\gamma_{I}^{s}(G)$, and this is a contradiction.

Case $3 x, y \in V_{2}$. Since $S_{x y} \neq \varnothing$ and $g$ is a $\gamma_{I}^{S}(G)$-function, $P_{g}(x)=\varnothing$ or $P_{g}(y)=\emptyset$. Hence, $\left|V_{g}\right|<\left|V_{2}\right|$ and, as in Case 2, we arrive to a contradiction.

Case $4 x \in V_{g}$. If $x \in P_{g}(y)$, then $S_{x y} \subseteq P_{g}(y)$ and $\left\langle P_{g}(y)\right\rangle$ is a clique, which is a contradiction. Now, if $y \in V_{g} \backslash\{x\}$, then $S_{x y}=\emptyset$, which is a contradiction. Finally, if $y \in V_{1} \cup V_{2}$ and $x \notin P_{g}(y)$, then $u, v \in P_{g}(y)$, which is a contradiction, as $\left\langle P_{g}(y)\right\rangle$ is a clique.

According to the four cases above, the proof of our claim is complete. Therefore, if for every $\gamma_{2}(G)$-set $S$ there exist two vertices $x, y \in S$ such that the set $S_{x y}$ contains 
two non-adjacent vertices, then $\gamma_{I}^{s}(G) \neq \gamma_{2}(G)$, and by Theorem 7 we conclude that $\gamma_{I}^{S}(G) \geq \gamma_{2}(G)+1$.

The bound above is tight. To see this we can take, for instance, the graph shown in Fig. 1, on the right. Now, in order to show that the converse of Theorem 9 does not hold, we can consider the graph shown in Fig. 1, on the left, where $\gamma_{I}^{S}(G)=5=\gamma_{2}(G)+1$ and the $\gamma_{2}(G)$-set $S$ consisting of all vertices of degree five satisfies that $\left\langle S_{x y}\right\rangle$ is a clique for every $x, y \in S$. Another example in the same direction is the graph sown in Fig. 2, where $\gamma_{I}^{s}(G)=7=\gamma_{2}(G)+1$ and the $\gamma_{2}(G)$-set $S$ consisting of all black-coloured vertices, except the central one, satisfies $S_{x y}=\emptyset$ for every $x, y \in S$.

\section{The particular case of cycles and trees}

Next we obtain closed formulas for the secure Italian domination number of cycles and paths. For this purpose, we shall need the following lemma.

Lemma 10 If $G \cong P_{n}$ or $G \cong C_{n}$, then there exists a $\gamma_{I}^{s}(G)$-function $f\left(V_{0}, V_{1}, V_{2}\right)$ such that $V_{2}=\emptyset$.

Proof It readily seen that the result follows for $n \leq 5$. Assume that $n \geq 6$. Let $f\left(V_{0}, V_{1}, V_{2}\right)$ be a $\gamma_{I}^{s}(G)$-function such that $\left|V_{2}\right|$ is minimum among the functions satisfying Lemma 1. Suppose that there exists $x \in V(G)$ such that $f(x)=2$. As assumed, $x$ is not a leaf. Let $N_{G}(x)=\{y, z\}$. If $f(y) \geq 1$, then we can construct a $\gamma_{I}^{S}(G)$-function $g\left(W_{0}, W_{1}, W_{2}\right)$ where $g(x)=1, g(z)=\max \{1, f(z)\}$ and $g(v)=$ $f(v)$ for every $v \in V(G) \backslash\{x, z\}$, which is a contradiction, as $\left|W_{2}\right|<\left|V_{2}\right|$. Hence, $f(y)=f(z)=0$. Let $\left\{y^{\prime}\right\}=N(y) \backslash\{x\}$ and $\left\{z^{\prime}\right\}=N(z) \backslash\{x\}$. Notice that $f\left(y^{\prime}\right) \geq 1$ or $f\left(z^{\prime}\right) \geq 1$, otherwise $f$ is not a $\gamma_{I}^{S}(G)$-function. Now, if $f\left(y^{\prime}\right) \geq 1$, then $x$ is a moving neighbour of $z$, and so we can construct a $\gamma_{I}^{s}(G)$-function $g\left(W_{0}, W_{1}, W_{2}\right)$ where $g(x)=g(z)=1$ and $g(v)=f(v)$ for every $v \in V(G) \backslash\{x, z\}$, which again is a contradiction, as $\left|W_{2}\right|<\left|V_{2}\right|$.

Proposition 11 For any integer $n \geq 3$,

$$
\gamma_{I}^{s}\left(C_{n}\right)=\left\lceil\frac{3 n}{5}\right\rceil .
$$

Proof The cases $n \in\{3,4\}$ are easy to check. Hence, from now on we assume that $n \geq 5$. Let $V\left(C_{n}\right)=\left\{v_{0}, v_{1}, \ldots, v_{n-1}\right\}$, where consecutive vertices are adjacent. First we show that $\gamma_{I}^{s}\left(C_{n}\right) \leq\left\lceil\frac{3 n}{5}\right\rceil$ by constructing a secure Italian dominating function $f\left(V_{0}, V_{1}, \emptyset\right)$ of weight $\omega(f)=\left\lceil\frac{3 n}{5}\right\rceil$. For $n \equiv i(\bmod 5)$ we define $l=\frac{n-i}{5}$ and consider a partition $\Pi_{l}$ of $V\left(C_{n}\right)$ defined as follows. If $i=0$, then $\Pi_{l}=\left\{X_{0}, \ldots, X_{l-1}\right\}$ and if $i \geq 1$, then $\Pi_{l}=\left\{X_{0}, \ldots, X_{l-1}, X_{l}\right\}$. For any $j<l$, the set $X_{j}=\left\{v_{5 j}, v_{5 j+1}, \ldots, v_{5 j+4}\right\}$ contains five consecutive vertices of $C_{n}$, while $X_{l}$ contains the remaining $i$ consecutive vertices.

For every $0 \leq j \leq l-1$, let $f\left(v_{5 j}\right)=1, f\left(v_{5 j+1}\right)=0, f\left(v_{5 j+2}\right)=1$, $f\left(v_{5 j+3}\right)=1, f\left(v_{5 i+4}\right)=0$. We can see these weights as $l$ consecutive sequences of numbers 10110 . The weight of the vertices in $X_{l}$ is assigned as follows. 
- If $n \equiv 1(\bmod 5)$, then $f\left(v_{n-1}\right)=1$.

- If $n \equiv 2(\bmod 5)$, then $f\left(v_{n-2}\right)=f\left(v_{n-1}\right)=1$.

- If $n \equiv 3(\bmod 5)$, then $f\left(v_{n-2}\right)=0$ and $f\left(v_{n-3}\right)=f\left(v_{n-1}\right)=1$.

- If $n \equiv 4(\bmod 5)$, then $f\left(v_{n-3}\right)=0$ and $f\left(v_{n-4}\right)=f\left(v_{n-2}\right)=f\left(v_{n-1}\right)=1$.

The weight of $f$ can be expressed in terms of $n \equiv i(\bmod 5)$ as follows.

- If $i=0$, then $\omega(f)=\frac{3 n}{5}=\left\lceil\frac{3 n}{5}\right\rceil$.

- If $i=1$ or $i=2$, the $\omega(f)=\frac{3(n-i)}{5}+i=\left\lceil\frac{3 n}{5}\right\rceil$.

- If $i=3$ or $i=4$, the $\omega(f)=\frac{3(n-i)}{5}+i-1=\left\lceil\frac{3 n}{5}\right\rceil$.

Notice that if $f(x)=0$ for $x \in V\left(C_{n}\right)$, then $x$ belongs to a sequence of consecutive vertices $a x b c$ with sequence of weights 1011, or belongs to a sequence of consecutive vertices $a b x c$ with sequence of weights 1101 . In both cases, $f_{b \rightarrow x}$ is an IDF. Hence, $f$ is an SIDF and so $\gamma_{I}^{s}\left(C_{n}\right) \leq \omega(f)=\left\lceil\frac{3 n}{5}\right\rceil$.

Now we show that $\gamma_{I}^{s}\left(C_{n}\right) \geq\left\lceil\frac{3 n}{5}\right\rceil$. Let $f\left(V_{0}, V_{1}, V_{2}\right)$ be a $\gamma_{I}^{s}\left(C_{n}\right)$-function which satisfies Lemma 10. Since $V_{2}=\emptyset$, we have that $V_{1}$ is a 2-dominating set, and so for any vertex $v_{i} \in V_{0}$, we have that $f\left(v_{i-1}\right)=f\left(v_{i+1}\right)=1$. Moreover, if $v_{i-1}$ is the moving neighbour of $v_{i}$, then $f\left(v_{i-2}\right)=1$, otherwise the moving neighbour of $v_{i}$ is $v_{i+1}$ and so $f\left(v_{i+2}\right)=1$. Thus, for every sequence of five consecutive vertices, at most two of them can belong to $V_{0}$, i.e.,

$\psi_{i}=f\left(v_{i-2}\right)+f\left(v_{i-1}\right)+f\left(v_{i}\right)+f\left(v_{i+1}\right)+f\left(v_{i+2}\right) \geq 3$ for every $i \in\{0, \ldots, n\}$.

Hence,

$$
5 \gamma_{I}^{s}\left(C_{n}\right)=5 \omega(f)=\sum_{i=0}^{n-1} \psi_{i} \geq 3 n
$$

Therefore, $\gamma_{I}^{s}\left(C_{n}\right) \geq\left\lceil\frac{3 n}{5}\right\rceil$.

Let $G$ be a graph and $e \in E(G)$. The edge-deletion subgraph $G-e$ of $G$ is defined to be $G-e=(V(G), E(G) \backslash\{e\})$. Observe that every $\gamma_{I}^{S}(G-e)$-function is an SIDF on $G$, which implies the following result.

Theorem 12 For any spanning subgraph $H$ of a graph $G$,

$$
\gamma_{I}^{S}(G) \leq \gamma_{I}^{S}(H)
$$

From Proposition 11 and Theorem 12 we derive the following consequence.

Theorem 13 For any Hamiltonian graph $G$ of order $n$,

$$
\gamma_{I}^{s}(G) \leq\left\lceil\frac{3 n}{5}\right\rceil .
$$

As an example of graph $G \nsubseteq C_{n}$ where the bound above is achieved, we take the graph shown in Fig. 1, on the left. 
Proposition 14 For any non-trivial path $P_{n}$,

$$
\gamma_{I}^{s}\left(P_{n}\right)=\left\lceil\frac{3 n+2}{5}\right\rceil \text {. }
$$

Proof The cases where $n \leq 4$ are easy to check. Hence, from now on we assume that $n \geq 5$. Let $f\left(V_{0}, V_{1}, V_{2}\right)$ be a $\gamma_{I}^{s}\left(P_{n}\right)$-function which satisfies Lemma 10 . In this proof we adapt the procedure developed in the proof of Proposition 11 to the case of paths. Thus, we assume that $V\left(P_{n}\right)=\left\{v_{0}, v_{1}, \ldots, v_{n-1}\right\}$, where consecutive vertices are adjacent. From Eq. (1) we know that $\psi_{i} \geq 3$ for every $i \in\{0, \ldots, n\}$. In this case, the weight of the leaves has to be one, i.e., $f\left(v_{0}\right)=f\left(v_{n-1}\right)=1$. Hence, the sequence $f\left(v_{0}\right) f\left(v_{1}\right) f\left(v_{2}\right) f\left(v_{3}\right)$ has to be 1011 or 1101 . Analogously, the sequence $f\left(v_{n-4}\right) f\left(v_{n-3}\right) f\left(v_{n-2}\right) f\left(v_{n-1}\right)$ has to be 1101 or 1011 . In all cases, $\psi_{1}=f\left(v_{3}\right)+f\left(v_{2}\right)+f\left(v_{1}\right)+f\left(v_{0}\right)+f\left(v_{n-1}\right) \geq 4$ and $\psi_{n-2}=f\left(v_{0}\right)+f\left(v_{n-1}\right)+$ $f\left(v_{n-2}\right)+f\left(v_{n-3}\right)+f\left(v_{n-4}\right) \geq 4$. Hence,

$$
5 \gamma_{I}^{s}\left(P_{n}\right)=5 \omega(f)=\sum_{i=0}^{n-1} \psi_{i} \geq 3 n+2
$$

which implies that $\gamma_{I}^{s}\left(P_{n}\right) \geq\left\lceil\frac{3 n+2}{5}\right\rceil$.

To conclude the proof we only need to construct an SIDF as described in the proof of Proposition 11, with the only difference that, for $n \equiv 0(\bmod 5)$ we take $f\left(v_{n-1}\right)=1$ and for $n \equiv 3(\bmod 5)$ we take $f\left(v_{n-2}\right)=1$. In this case, the weight of $f$ can be expressed as follows.

- If $n \equiv 0(\bmod 5)$, then $\omega(f)=\frac{3 n}{5}+1=\left\lceil\frac{3 n+2}{5}\right\rceil$.

- If $n \equiv 1(\bmod 5)$, the $\omega(f)=\frac{3(n-1)}{5}+1=\left\lceil\frac{3 n+2}{5}\right\rceil$.

- If $n \equiv 2(\bmod 5)$, the $\omega(f)=\frac{3(n-2)}{5}+2=\left\lceil\frac{3 n+2}{5}\right\rceil$.

- If $n \equiv 3(\bmod 5)$, the $\omega(f)=\frac{3(n-3)}{5}+3=\left\lceil\frac{3 n+2}{5}\right\rceil$.

- If $n \equiv 4(\bmod 5)$, the $\omega(f)=\frac{3(n-4)}{5}+3=\left\lceil\frac{3 n+2}{5}\right\rceil$.

Therefore, $\gamma_{I}^{s}\left(P_{n}\right) \leq \omega(f)=\left\lceil\frac{3 n+2}{5}\right\rceil$.

From Remark 8 (c) we learned that $\gamma_{I}^{S}(G) \geq \gamma(G)+1$ for every non-empty graph $G$. We proceed to characterize the trees $T$ satisfying the equalities $\gamma_{I}^{S}(T)=\gamma(T)+1$ or $\gamma_{I}^{S}(T)=\gamma(T)+2$. We begin with the following observation.

Observation 15 If $T$ is a tree of order $n \geq 3$, then always is possible to choose a $\gamma(T)$-set not containing any leaf of $T$.

From Observation 15 and Lemma 1 we have the following lemma.

Lemma 16 Let $T$ be a tree. If $\gamma_{I}^{S}(T)=\gamma(T)+1$, there there exists at most one strong support $s$ in $T$ and $s$ is the neighbour of exactly two leaves.

We are now ready to prove the following characterization. 
Theorem $17 \gamma_{I}^{S}(T)=\gamma(T)+1$ if and only if $T \cong P_{2}$ or $T \cong P_{4}$.

Proof If $T \cong P_{2}$ or $T \cong P_{4}$, then obviously $\gamma_{I}^{s}(T)=\gamma(T)+1$.

Let $n$ be the order of $T$. If $n \leq 4$, then the only trees with $\gamma_{I}^{S}(T)=\gamma(T)+1$ are $P_{2}$ and $P_{4}$, as desired. From now on, suppose that $T$ is a tree of minimum order $n \geq 5$ among the trees satisfying the equality $\gamma_{I}^{S}(T)=\gamma(T)+1$. Obviously, if $T^{\prime}$ is a tree of order less than $n$ such that $\gamma_{I}^{s}\left(T^{\prime}\right)=\gamma\left(T^{\prime}\right)+1$, then $T^{\prime}$ is either $P_{2}$ or $P_{4}$. Let $f$ be a $\gamma_{I}^{S}(T)$-function satisfying Lemma 1 . Let $P=\left(v_{0}, \ldots, v_{l}\right)$ be a longest path in $T$. If $l \leq 2$, then $T \cong K_{1, n-1}$, which is contradiction with Lemma 16 . Thus, $l \geq 3$. Assume first that $\mathrm{d}_{T}\left(v_{1}\right)>2$. Then, from Lemma $16, \mathrm{~d}_{T}\left(v_{1}\right)=3$, and so there exist two leaves $x, v_{0}$ adjacent to $v_{1}$. Let $T_{1}=T-\left\{v_{0}, x, v_{1}\right\}$. Since $f\left(v_{0}\right)=f(x)=1$ and $f\left(v_{1}\right)+f\left(v_{2}\right) \geq 1$, we deduce that $\gamma_{I}^{s}\left(T_{1}\right)+2 \leq \gamma_{I}^{s}(T)=\gamma(T)+1 \leq \gamma\left(T_{1}\right)+1+1$, what gives $\gamma_{I}^{S}\left(T_{1}\right) \leq \gamma\left(T_{1}\right)$, which is a contradiction with Remark 8 (c). Hence, $\mathrm{d}_{T}\left(v_{1}\right)=2$. Now, let $T_{2}=T-\left\{v_{0}, v_{1}\right\}$. Since $f\left(v_{0}\right)=1$ and $f\left(v_{1}\right)+f\left(v_{2}\right) \geq 1$, we deduce that $\gamma_{I}^{S}\left(T_{2}\right)+1 \leq \gamma_{I}^{S}(T)=\gamma(T)+1 \leq \gamma\left(T_{2}\right)+1+1$, what gives $\gamma_{I}^{s}\left(T_{2}\right) \leq \gamma\left(T_{2}\right)+1$. Thus, Remark 8 (c) leads to $\gamma_{I}^{s}\left(T_{2}\right)=\gamma\left(T_{2}\right)+1$, and by assumption $T_{2} \cong P_{2}$ or $T_{2} \cong P_{4}$. If $T_{2} \cong P_{2}$, then $T \cong P_{4}$, which is a contradiction, as $n \geq 5$. If $T_{2} \cong P_{4}$, then $T \cong P_{6}$ or $T \cong P_{3} \odot K_{1}$, but in both cases $\gamma_{I}^{S}(T)>\gamma(T)+1$, which is a contradiction again.

From the result above we conclude that if $T \not P_{2}$ and $T \not P_{4}$, then $\gamma_{I}^{s}(T) \geq$ $\gamma(T)+2$. Below we give the full characterization of the extremal trees. First we define a family $\mathcal{R}$ of trees. A spider is a graph obtained from the star $K_{1, t}$ for $t \geq 1$ by subdividing each edge of the star exactly once. Obviously, if $T$ is a spider with $t=1$, then $T \cong P_{3}$. If $t \geq 3$ and we subdivide once exactly $t-1$ of the edges of a star $K_{1, t}$, then we obtain a tree called slightly wounded spider. We say that $T \in \mathcal{R}$ if $T$ is a spider, a slightly wounded spider with $t \geq 3$, a path $P_{6}$, a path $P_{7}$, a corona $P_{4} \odot K_{1}$, a star $K_{1,3}$ with one subdivided edge (we denote it by $T_{*}$ ) or a star $K_{1,3}$ with one edge subdivided once and one edge subdivided twice (for simplicity we denote it by $T_{* *}$ ).

Theorem 18 Let $T$ be a tree. Then $\gamma_{I}^{S}(T)=\gamma(T)+2$ if and only if $T \in \mathcal{R}$.

Proof If $T \in \mathcal{R}$, then obviously $\gamma_{I}^{S}(T)=\gamma(T)+2$. To prove the converse, we use induction on $n$, the number of vertices of a tree. The smallest tree such that $\gamma_{I}^{S}(T)=\gamma(T)+2$ is $T \cong P_{3}$, which is a spider, so $T \in \mathcal{R}$. Assume that $n \geq 4$ and if $T^{\prime}$ is a tree with $\left|V\left(T^{\prime}\right)\right|<n$ and $\gamma_{I}^{s}\left(T^{\prime}\right)=\gamma\left(T^{\prime}\right)+2$, then $T^{\prime} \in \mathcal{R}$.

Let $T$ be a tree of order $n \geq 4$ such that $\gamma_{I}^{S}(T)=\gamma(T)+2$, and let $f$ be a $\gamma_{I}^{S}(T)$ function which satisfies Lemma 1 and the values of $f$ on supports on the longest paths is as small as possible. Notice that $f(x)=0$ for every support $x$ of degree two. Let $P=\left(v_{0}, \ldots, v_{l}\right)$ be a longest path in $T$. We differentiate some cases according to the degree of $v_{1}$ and $v_{2}$.

Case $1 \mathrm{~d}_{T}\left(v_{1}\right)>2$. Let $T^{\prime}=T-\left\{v_{0}\right\}$. Since $v_{1}$ is a support of $T^{\prime}$, the restriction of $f$ to $T^{\prime}$ is an SIDF. Thus, $\gamma_{I}^{s}\left(T^{\prime}\right) \leq \gamma_{I}^{s}(T)-1$ and $\gamma\left(T^{\prime}\right)=\gamma(T)$. From our assumption, $\gamma_{I}^{s}(T)=\gamma(T)+2$, which implies that $\gamma_{I}^{s}\left(T^{\prime}\right)=\gamma\left(T^{\prime}\right)+1$. By Theorem 17, $T^{\prime} \cong P_{2}$ or $T^{\prime} \cong P_{4}$. And since $\mathrm{d}_{T}\left(v_{1}\right)>2$, the only possibility is $T^{\prime} \cong P_{4}$, where $v_{1}$ is a support of $P_{4}$. This implies that $T \cong T_{*} \in \mathcal{R}$.

Case $2 \mathrm{~d}_{T}\left(v_{1}\right)=2$. Let $T^{\prime}=T-\left\{v_{0}, v_{1}\right\}$. From the choice of $f$ we have $f\left(v_{0}\right)=1$ and $f\left(v_{1}\right)=0$. Thus, the restriction of $f$ to $T^{\prime}$ is an SIDF, which implies 
that $\gamma_{I}^{s}\left(T^{\prime}\right) \leq \gamma_{I}^{s}(T)-1=\gamma(T)+1$. Moreover, $\gamma\left(T^{\prime}\right)+1 \leq \gamma_{I}^{s}\left(T^{\prime}\right)$, by Remark 8 . Hence,

$$
\gamma\left(T^{\prime}\right)+1 \leq \gamma_{I}^{S}\left(T^{\prime}\right) \leq \gamma(T)+1 .
$$

With this facts in mind, we consider the following three subcases, depending on the degree of vertex $v_{2}$.

Subcase $2.1 \mathrm{~d}_{T}\left(v_{2}\right)>2$ and there exists a vertex $s \in N_{T}\left(v_{2}\right) \backslash\left\{v_{1}, v_{3}\right\}$ which is a support. Since $s \in N_{T}\left(v_{2}\right)$ is a support, $\gamma\left(T^{\prime}\right)=\gamma(T)-1$. Hence, by Eq. (2) we have that $\gamma\left(T^{\prime}\right)+1 \leq \gamma_{I}^{s}\left(T^{\prime}\right) \leq \gamma(T)+1=\gamma\left(T^{\prime}\right)+2$, and so either $\gamma_{I}^{s}\left(T^{\prime}\right)=\gamma\left(T^{\prime}\right)+1$ or $\gamma_{I}^{s}\left(T^{\prime}\right)=\gamma\left(T^{\prime}\right)+2$. Observe that since there exists a vertex $s \in N_{T}\left(v_{2}\right) \backslash\left\{v_{1}, v_{3}\right\}$ which is a support vertex, it follows that $\left|V\left(T^{\prime}\right)\right| \geq 5$ (because of the diametral path P). Using this fact, we deduce that $\gamma_{I}^{S}\left(T^{\prime}\right)=\gamma\left(T^{\prime}\right)+1$ cannot occur. So, if $\gamma_{I}^{S}\left(T^{\prime}\right)=\gamma\left(T^{\prime}\right)+2$, then by induction hypothesis we have that $T^{\prime} \in \mathcal{R}$. If $T^{\prime}$ is a spider or a slightly wounded spider, then $T$ is also a spider or a slightly wounded spider, respectively, so $T \in \mathcal{R}$. Since $s \notin\left\{v_{1}, v_{3}\right\}, T^{\prime} \not T_{*}$ and, in the remaining cases it is easy to check that $\gamma_{I}^{S}(T) \geq \gamma(T)+3$, which is a contradiction.

Subcase $2.2 \mathrm{~d}_{T}\left(v_{2}\right)>2$ and every vertex in $N_{T}\left(v_{2}\right) \backslash\left\{v_{1}, v_{3}\right\}$ is a leaf. Since $v_{2}$ is a support in $T^{\prime}, \gamma\left(T^{\prime}\right)=\gamma(T)-1$. Thus, as in Subcase 2.1, either $\gamma_{I}^{S}\left(T^{\prime}\right)=\gamma\left(T^{\prime}\right)+1$ or $\gamma_{I}^{s}\left(T^{\prime}\right)=\gamma\left(T^{\prime}\right)+2$. If $\gamma_{I}^{s}\left(T^{\prime}\right)=\gamma\left(T^{\prime}\right)+1$, then from Theorem 17 and from the fact that $\mathrm{d}_{T^{\prime}}\left(v_{2}\right) \geq 2$, we deduce that $T^{\prime}=P_{4}$ and $T$ is a slightly wounded spider, so $T \in \mathcal{R}$. Now, if $\gamma_{I}^{s}\left(T^{\prime}\right)=\gamma\left(T^{\prime}\right)+2$, then by induction hypothesis, $T^{\prime} \in \mathcal{R}$. If $T^{\prime}$ is a spider with $t=1$, then $T=T_{*} \in \mathcal{R}$. If $T^{\prime}$ is a slightly wounded spider with $t=3$, then $T=P_{4} \odot K_{1} \in \mathcal{R}$. In the remaining cases it is easy to check that $\gamma_{I}^{S}(T) \geq \gamma(T)+3$, which is a contradiction.

Subcase $2.3 \mathrm{~d}_{T}\left(v_{2}\right)=2$. Obviously, $\gamma\left(T^{\prime}\right) \geq \gamma(T)-1$. Thus, by Eq. (2), $\gamma\left(T^{\prime}\right)+$ $1 \leq \gamma_{I}^{s}\left(T^{\prime}\right) \leq \gamma\left(T^{\prime}\right)+2$. By Theorem 17, if $\gamma_{I}^{s}\left(T^{\prime}\right)=\gamma\left(T^{\prime}\right)+1$, then $T^{\prime} \cong P_{2}$ or $T^{\prime} \cong P_{4}$. In the first case, $T \cong P_{4}$, which is a contradiction, as $\gamma_{I}^{s}\left(P_{4}\right)=3 \neq$ $\gamma\left(P_{4}\right)+2$. In the second case $T \cong P_{6} \in \mathcal{R}$. Now, if $\gamma_{I}^{s}\left(T^{\prime}\right)=\gamma\left(T^{\prime}\right)+2$, then by induction hypothesis, $T^{\prime} \in \mathcal{R}$. If $T^{\prime}$ is a spider with $t=2$, then $T \cong P_{7} \in \mathcal{R}$. If $T^{\prime} \cong T_{*}$ and $v_{2}$ is a leaf of $T^{\prime}$ adjacent to a central vertex, then $T \cong T_{* *} \in \mathcal{R}$. In the remaining cases it is easy to check that $\gamma_{I}^{S}(T) \geq \gamma(T)+3$, which is a contradiction.

\section{The particular case of join graphs}

To begin this section we consider the class of join graphs of the form $K_{n}+G$.

Theorem 19 For any non-complete graph $G$ and any integer $n \geq 2$, the following statements hold.

(i) $3 \leq \gamma_{I}^{s}\left(K_{1}+G\right) \leq \min \left\{\gamma(G)+2, \gamma_{r}(G)+1\right\}$.

(ii) $\gamma_{I}^{s}\left(K_{n}+G\right)=3$.

Proof Since $G$ is a non-complete graph, $K_{1}+G$ is a non-complete graph, and so Theorem 5 leads to $\gamma_{I}^{s}\left(K_{1}+G\right) \geq 3$.

Now, let $S$ be a $\gamma(G)$-set. It is immediate that the function $f\left(V_{0}, V_{1}, V_{2}\right)$, defined on $K_{1}+G$ by $V_{2}=V\left(K_{1}\right)$ and $V_{1}=S$, is an SIDF, which implies that $\gamma_{I}^{s}\left(K_{1}+\right.$ 
$G) \leq \omega(f)=\gamma(G)+2$. To conclude the proof of (i) we take a $\gamma_{r}(G)$-function $g\left(X_{0}, X_{1}, X_{2}\right)$ and define a function $h\left(Y_{0}, Y_{1}, Y_{2}\right)$ on $K_{1}+G$ by $Y_{1}=X_{1} \cup V\left(K_{1}\right)$ and $V_{2}=X_{2}$. Obviously, $h$ is an SIDF and so $\gamma_{I}^{s}\left(K_{1}+G\right) \leq \omega(h)=\gamma_{r}(G)+1$.

Finally, if $n \geq 2$, then $K_{n}+G=K_{1}+\left(K_{n-1}+G\right)$. Since $\gamma\left(K_{1}+G\right)=1$, from (i) we deduce (ii).

From the result above we deduce the following corollary.

Corollary 20 If $\gamma_{r}(G)=2$, then $\gamma_{I}^{s}\left(K_{1}+G\right)=3$.

Next we consider the class of join graphs $G+H$ where neither $G$ nor $H$ is a complete graph.

Theorem 21 For any non-complete graphs $G$ and $H$,

$$
3 \leq \gamma_{I}^{s}(G+H) \leq \min \left\{6, \gamma_{I}^{s}(G), \gamma_{I}^{s}(H)\right\}
$$

Proof The lower bound is a consequence of Theorem 5, as $G+H$ is a non-complete graph. Now, since $G$ and $H$ are non-trivial graphs, we can construct a function $f\left(V_{0}, V_{1}, V_{2}\right)$ on $G+H$ by taking two vertices $u, v \in V(G)$ and two vertices $x, y \in V(H)$, and defining $V_{2}=\{u, x\}$ and $V_{1}=\{v, y\}$. It is readily seeing that $f$ is an SIDF, and so $\gamma_{I}^{S}(G+H) \leq \omega(f)=6$. To conclude the proof we only need to observe that $\gamma_{I}^{s}(G) \geq 3$ and so, from any $\gamma_{I}^{s}(G)$-function $g\left(X_{0}, X_{1}, X_{2}\right)$, we can construct an SIDF $g^{\prime}\left(Y_{0}, Y_{1}, Y_{2}\right)$ on $G+H$ by $Y_{0}=X_{0} \cup V(H), Y_{1}=X_{1}$ and $Y_{2}=X_{2}$. Therefore, $\gamma_{I}^{s}(G+H) \leq \omega\left(g^{\prime}\right)=\gamma_{I}^{s}(G)$.

Now we consider some particular cases.

Theorem 22 The following statements hold.

(a) If $\gamma_{I}^{s}(G)=3$, then $\gamma_{I}^{s}(G+H)=3$ for every graph $H$.

(b) If $\gamma(G)=\gamma(H)=2$, then $3 \leq \gamma_{I}^{s}(G+H) \leq 4$.

(c) If $\gamma_{r}(G)=2$ and $\gamma_{r}(H)=2$, then $\gamma_{I}^{s}(G+H)=3$.

(d) If $\gamma_{r}(G) \geq 3$ and $H$ is a non-complete graph, then $3 \leq \gamma_{I}^{s}(G+H) \leq$ $\min \left\{6, \gamma_{r}(G)+1\right\}$.

Proof If $H$ is a non-complete graph, then we deduce (a) from Theorem 21. Now, if $H \cong K_{n}, n \geq 2$ then we apply Theorem 19 (ii), while for $H \cong K_{1}$ we apply Theorems 7 and 19 (i).

In the case of item (b), we only need to observe that for any $\gamma(G)$-set $S$ and any $\gamma(H)$-set $S^{\prime}$ we can define an $\operatorname{SIDF} f\left(V_{0}, V_{1}, V_{2}\right)$ on $G+H$, as $V_{1}=S \cup S^{\prime}$ and $V_{2}=\emptyset$. Thus, $3 \leq \gamma_{I}^{s}(G+H) \leq \omega(f)=4$.

Now, assume that $\gamma_{r}(G)=2$ and $\gamma_{r}(H)=2$. Let $v \in V(H)$ be a vertex of a positive weight under any $\gamma_{r}(H)$-function. Let $f^{\prime}\left(W_{0}, W_{1}, W_{2}\right)$ be a $\gamma_{r}(G)$-function. We define a function $f^{\prime \prime}\left(U_{0}, U_{1}, U_{2}\right)$ on $G+H$ by $U_{1}=W_{1} \cup\{v\}$ and $U_{2}=W_{2}$. Notice that either $v$ is a universal vertex of $H$ or $\langle V(H) \backslash N[v]\rangle$ is a clique. Hence, $f^{\prime \prime}$ is an SIDF on $G+H$. Therefore, $3 \leq \gamma_{I}^{S}(G+H) \leq \omega\left(f^{\prime \prime}\right)=3$, concluding that (c) follows. 

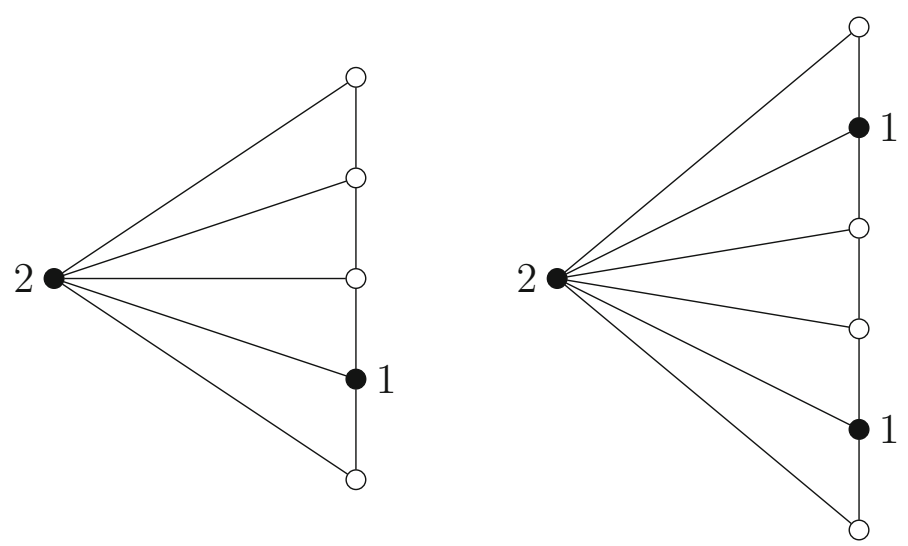

Fig. $3 \gamma_{I}^{s}\left(K_{1}+P_{5}\right)=3$ and $\gamma_{I}^{s}\left(K_{1}+P_{6}\right)=4$

To conclude the proof, we take one vertex $v \in V(H)$, a $\gamma_{r}(G)$-function $g\left(X_{0}, X_{1}, X_{2}\right)$ and define a function $g^{\prime}\left(Y_{0}, Y_{1}, Y_{2}\right)$ on $G+H$ by $Y_{1}=X_{1} \cup\{v\}$ and $V_{2}=X_{2}$. Obviously, if $\gamma_{r}(G) \geq 3$, then $g^{\prime}$ is an SIDF and so $\gamma_{I}^{s}(G+H) \leq$ $\omega\left(g^{\prime}\right)=\gamma_{r}(G)+1$. Therefore, by Theorem 21 we conclude that (d) follows.

Since $\gamma_{r}(G)=2$ if and only if $G$ is a non-complete graph with $\gamma(G)=1$ or $\gamma_{s}(G)=2$, from Theorem 22 (c) and Corollary 20 we deduce the following result.

Remark 23 The following statements hold.

(a) If $\gamma(G)=\gamma(H)=1$ and $\left(H \not K_{n}\right.$ or $\left.G \not K_{n^{\prime}}\right)$, then $\gamma_{I}^{S}(G+H)=3$.

(b) If $\gamma(G)=1$ and $\gamma_{s}(H)=2$, then $\gamma_{I}^{s}(G+H)=3$.

(c) If $\gamma_{s}(G)=2$ and $\gamma_{s}(H)=2$, then $\gamma_{I}^{s}(G+H)=3$.

Next we consider the classes of fan and wheel graphs.

The Italian domination number and weak Roman domination number of a cycle $C_{n}$, where $n \geq 4$, was determined in Chellali et al. (2016) and in Henning and Hedetniemi (2003), respectively.

Lemma 24 For $n \geq 4$,

1. (Chellali et al. 2016) $\gamma_{I}\left(C_{n}\right)=\left\lceil\frac{n}{2}\right\rceil$,

2. (Henning and Hedetniemi 2003) $\gamma_{r}\left(C_{n}\right)=\left\lceil\frac{3 n}{7}\right\rceil$.

Proposition 25 Let $n \geq 4$ be an integer. For the classes of fan graphs $K_{1}+P_{n}$ and wheel graphs $K_{1}+C_{n}$,

$$
\gamma_{I}^{s}\left(K_{1}+P_{n}\right)=2+\left\lceil\frac{n-2}{3}\right\rceil \text { and } \gamma_{I}^{s}\left(K_{1}+C_{n}\right)=2+\left\lceil\frac{n-2}{3}\right\rceil
$$

Proof The cases $n \in\{4,5,6\}$ are easy to check. Hence, from now on we assume that $n \geq 7$. In Fig. 3 we show examples of $\gamma_{I}^{s}\left(K_{1}+P_{n}\right)$-functions for $n=5$ and $n=6$. 
Let $P_{n}=\left(x_{1}, \ldots, x_{n}\right)$ and let $S$ be a $\gamma\left(P_{n-2}\right)$-set, where $P_{n-2}$ is obtained from $P_{n}$ by removing $x_{n-1}$ and $x_{n}$. We can construct an SIDF $g$ on $K_{1}+P_{n}$ by assigning the label 1 to every vertex in $S$, label 2 to the vertex of $K_{1}$ and 0 to the remaining vertices. Hence, $\gamma_{I}^{s}\left(K_{1}+P_{n}\right) \leq \omega(g)=2+\gamma\left(P_{n-2}\right)=2+\left\lceil\frac{n-2}{3}\right\rceil$.

In order to show that $\gamma_{I}^{s}\left(K_{1}+C_{n}\right) \geq 2+\left\lceil\frac{n-2}{3}\right\rceil$, let $f$ be a $\gamma_{I}^{s}\left(K_{1}+C_{n}\right)$-function, $f^{\prime}$ the restriction of $f$ to $C_{n}$ and $V\left(K_{1}\right)=\{v\}$. We differentiate three cases.

Case $1 f(v)=0$. In this case $f^{\prime}$ is an SIDF on $C_{n}$ and so $\gamma_{I}^{s}\left(K_{1}+C_{n}\right)=\omega(f)=$ $\omega\left(f^{\prime}\right) \geq \gamma_{I}^{s}\left(C_{n}\right) \geq \gamma_{I}\left(C_{n}\right)=\left\lceil\frac{n}{2}\right\rceil \geq 2+\left\lceil\frac{n-2}{3}\right\rceil$ for $n \geq 7$.

Case $2 f(v)=1$. If $f^{\prime}$ is a WRDF on $C_{n}$, then using Lemma $24 \gamma_{I}^{s}\left(K_{1}+C_{n}\right)=$ $\omega(f)=1+\omega\left(f^{\prime}\right) \geq 1+\gamma_{r}\left(C_{n}\right)=1+\left\lceil\frac{3 n}{7}\right\rceil \geq 2+\left\lceil\frac{n-2}{3}\right\rceil$. Now, if $f^{\prime}$ is not a WRDF on $C_{n}$, then there exists $u \in V\left(C_{n}\right)$ such that $f_{v \rightarrow u}$ is an IDF on $C_{n}$. Hence, using Lemma $24 \gamma_{I}^{s}\left(K_{1}+C_{n}\right)=\omega(f)=1+\omega\left(f^{\prime}\right) \geq \gamma_{I}\left(C_{n}\right)=\left\lceil\frac{n}{2}\right\rceil \geq 2+\left\lceil\frac{n-2}{3}\right\rceil$ for $n \geq 7$.

Case $3 f(v)=2$. If $f\left(N_{C_{n}}[x]\right)>0$ for every $x \in V\left(C_{n}\right)$, then $\gamma_{I}^{s}\left(K_{1}+C_{n}\right)=$ $\omega(f) \geq 2+\gamma\left(C_{n}\right)=2+\left\lceil\frac{n}{3}\right\rceil>2+\left\lceil\frac{n-2}{3}\right\rceil$. Now, if there exists exactly one vertex $x \in V\left(C_{n}\right)$ such that $f\left(N_{C_{n}}[x]\right)=0$, then $\gamma_{I}^{s}\left(K_{1}+C_{n}\right)=\omega(f) \geq 2+\gamma\left(P_{n-1}\right)=$ $2+\left\lceil\frac{n-1}{3}\right\rceil \geq 2+\left\lceil\frac{n-2}{3}\right\rceil$. Finally, suppose that there exist two vertices $x, y \in V\left(C_{n}\right)$ such that $f\left(N_{C_{n}}[x]\right)=f\left(N_{C_{n}}[y]\right)=0$. In such a case, $x$ and $y$ have to be adjacent, otherwise neither $f_{v \rightarrow x}$ nor $f_{v \rightarrow y}$ is an IDF on $K_{1}+C_{n}$, which is a contradiction. Hence, $\gamma_{I}^{s}\left(K_{1}+C_{n}\right)=\omega(f) \geq 2+\gamma\left(P_{n-2}\right)=2+\left\lceil\frac{n-2}{3}\right\rceil$.

According to the three cases above we conclude that $\gamma_{I}^{s}\left(K_{1}+C_{n}\right) \geq 2+\left\lceil\frac{n-2}{3}\right\rceil$.

Finally, since $K_{1}+P_{n}$ is a spanning subgraph of $K_{1}+C_{n}$, by Theorem 12 we have that $\gamma_{I}^{s}\left(K_{1}+C_{n}\right) \leq \gamma_{I}^{s}\left(K_{1}+P_{n}\right)$. Therefore, the result follows.

Open Access This article is licensed under a Creative Commons Attribution 4.0 International License, which permits use, sharing, adaptation, distribution and reproduction in any medium or format, as long as you give appropriate credit to the original author(s) and the source, provide a link to the Creative Commons licence, and indicate if changes were made. The images or other third party material in this article are included in the article's Creative Commons licence, unless indicated otherwise in a credit line to the material. If material is not included in the article's Creative Commons licence and your intended use is not permitted by statutory regulation or exceeds the permitted use, you will need to obtain permission directly from the copyright holder. To view a copy of this licence, visit http://creativecommons.org/licenses/by/4.0/.

\section{References}

Burger AP, Henning MA, van Vuuren JH (2008) Vertex covers and secure domination in graphs. Quaest Math 31(2):163-171

Chellali M, Haynes TW, Hedetniemi ST (2014) Bounds on weak Roman and 2-rainbow domination numbers. Discrete Appl Math 178:27-32

Chellali M, Haynes TW, Hedetniemi ST, McRae AA (2016) Roman 2-domination. Discrete Appl Math 204:22-28

Cockayne EJ, Favaron O, Mynhardt CM (2003) Secure domination, weak Roman domination and forbidden subgraphs. Bull Inst Comb Appl 39:87-100

Cockayne EJ, Dreyer PA Jr, Hedetniemi SM, Hedetniemi ST (2004) Roman domination in graphs. Discrete Math 278(1-3):11-22

Cockayne EJ, Grobler PJP, Gründlingh WR, Munganga J, van Vuuren JH (2005) Protection of a graph. Util Math 67:19-32 
Haynes T, Hedetniemi S, Slater P (1998a) Domination in graphs: volume 2: advanced topics. Chapman \& Hall/CRC Pure and Applied Mathematics, Taylor \& Francis, Routledge

Haynes TW, Hedetniemi ST, Slater PJ (1998b) Fundamentals of domination in graphs. Chapman and Hall/CRC Pure and Applied Mathematics Series, Marcel Dekker, Inc., New York

Henning MA, Hedetniemi ST (2003) Defending the Roman Empire-a new strategy. Discrete Math 266(13):239-251 (the 18th British Combinatorial Conference (Brighton, 2001))

Henning MA, Klostermeyer WF (2017) Italian domination in trees. Discrete Appl Math 217:557-564

Klostermeyer WF, MacGillivray G (2019) Roman, Italian, and 2-domination. J Comb Math Comb Comput 108:125-146

Klostermeyer WF, Mynhardt CM (2008) Secure domination and secure total domination in graphs. Discuss Math Graph Theory 28(2):267-284

Merouane H, Chellali M (2015) On secure domination in graphs. Inf Process Lett 115(10):786-790

Stewart I (1999) Defend the Roman empire!. Sci Am 281(6):136-138

Valveny M, Rodríguez-Velázquez JA (2019) Protection of graphs with emphasis on cartesian product graphs. Filomat 33:319-333

Valveny M, Pérez-Rosés H, Rodríguez-Velázquez JA (2019) On the weak Roman domination number of lexicographic product graphs. Discrete Appl Math 263:257-270

Publisher's Note Springer Nature remains neutral with regard to jurisdictional claims in published maps and institutional affiliations. 